\title{
Comparação dos valores de irregularidade longitudinal medidos com o perfilômetro a laser com variação do arranjo das posições dos sensores
}

\author{
Sérgio Armando de Sá e Benevides ${ }^{1}$; Laura Maria Goretti da Motta ${ }^{2}$
}

\begin{abstract}
Resumo: Este estudo tem como objetivo analisar a correlação entre os valores de irregularidade longitudinal efetuados com o perfilômetro inercial a laser com 3 sensores dispostos em 3 posições diferentes, em rodovias estaduais do Ceará. Na posição normal foram selecionados 72 trechos na Região Metropolitana de Fortaleza (RMF), dentre estes trechos iniciais foram selecionados 42 com os sensores dispostos no lado direito (LD) e no lado esquerdo (LE). O Software Ciberlogger foi utilizado para a coleta dos dados e para a determinação dos perfis longitudinais dos pavimentos, enquanto que o Software Cibershell foi usado para o cálculo do Índice de Irregularidade longitudinal (IRI). Nas equações de regressão estabelecidas, foram utilizados os programas Microsoft Office Excel e SPSS (Statistical Package for the Social Sciences), usando a Análise da Variância (ANOVA). Os resultados mostram que há correlação entre os valores do IRI medidos nas trilhas interna e externa, IRI $_{\text {int }} \times$ IRI $_{\text {ext }}$, obtidos com a localização dos sensores na posição normal. As análises mostram também que há correlação entre estes mesmos valores e os obtidos com os sensores dispostos no LD e LE.
\end{abstract}

\begin{abstract}
The purpose of this study is to analyze the correlation between the values of the International Roughness Index - IRI, measured with an inertial laser profilometer with 3 sensors in 3 different positions. The pavements investigated were from roads of the state of Ceará. In the normal position, seventy two sections of the state road network were selected, from Fortaleza Metropolitan Region (RMF), among these, 42 were selected with the sensors placed in the right (LD) and in left (LE) side. The Softwares Ciberlogger and Cibershell were used for data collection and for determining the longitudinal road profile The programs Microsoft Office Excel and SPSS (Statistical Package for the Social Sciences) were used for the regression equations and analysis of variance. The results indicate a correlation between IRI values measured from the inbound and outbound wheel path, obtained with the sensors located in normal position. The analysis also suggests a correlation between these values and results obtained with the sensors placed in LD and LE.
\end{abstract}

\section{INTRODUÇÃO}

O Sistema de Gerência de Pavimento (SGP) tem como objetivo principal a utilização de informações confiáveis e critérios de decisão que possibilitem o estabelecimento de um programa de construção, manutenção e reabilitação de pavimentos, dando o máximo retorno possível para os recursos disponíveis. Para alcançar este objetivo, o SGP deve ser capaz de comparar, priorizar e alocar os recursos do programa de manutenção e reabilitação (M\&R).

Os pavimentos podem, por diversas razões, apresentar uma gama variada de defeitos que por sua vez, afetam a serventia, as capacidades funcionais e estruturais dos pavimentos, causando desconforto aos usuários (SHRP, 1993). A avaliação das condições funcionais e estruturais de um pavimento deve ser efetuada por procedimentos adequados às suas características, utilizando-se várias metodologias para levantamento de defeitos que surgem na superfície dos pavimentos. O clima, a solicitação do tráfego, as características dos

\footnotetext{
${ }^{1}$ Sérgio Armando de Sá e Benevides, Programa de Engenharia de Transportes - COPPE, Universidade Federal do Rio de Janeiro, Rio de Janeiro, RJ, Brasil. (e-mail: sergio@det.ufc.br).

${ }^{2}$ Laura Maria Goretti da Motta, Programa de Engenharia de Transportes - COPPE, Universidade Federal do Rio de Janeiro, Rio de Janeiro, RJ, Brasil. (e-mail: laura@coc.ufrj.br).
}

Manuscrito recebido em 7/12/2009 e aprovado para publicação em 10/3/2010. Este artigo é parte de TRANSPORTES, volume XVIII, número 2, junho de 2010. ISSN: 2237-1346 (online). materiais e os processos construtivos, são fatores que atuam de forma isolada ou simultânea, são as causas destes defeitos.

As condições superficiais de um pavimento podem ser avaliadas de forma subjetiva, através de índices de serventia, ou objetiva, como, por exemplo, através da irregularidade longitudinal. Este parâmetro é internacionalmente utilizado, sendo medido por uma escala de referência conhecida como IRI - Índice de Irregularidade Internacional (International Roughness Index).

Medina (1997) destaca que a avaliação da condição funcional da superfície do pavimento permite que sejam estimados o conforto e a segurança que estão sendo oferecidos aos usuários. A estimativa pode ser feita de forma subjetiva, utilizando-se pessoas que transitam pelo trecho e dão notas, por exemplo, de zero (0) a cinco (5), associando assim a nota subjetiva aos defeitos. Mas uma avaliação objetiva é o desejável, especialmente com uso de equipamentos que permitam rapidez de aquisição de dados para fins de gestão dos recursos de manutenção disponíveis para uma rede de estradas.

A irregularidade longitudinal é um defeito de classe funcional associado à qualidade de rolamento e à segurança do pavimento. Haas et al. (1994) citam que a dinâmica dos veículos é afetada pelas distorções na superfície, e que estas causam desgaste, acréscimos no tempo de viagem e na manutenção. Os movimentos e esforços indesejáveis gerados nos veículos pela irre- 
gularidade longitudinal levam a uma condição de rolamento desconfortável, insegura e anti-econômica.

Esta pesquisa tem como objetivo verificar se os valores do IRI obtidos com o perfilômetro a laser com 3 sensores dispostos em 3 posições diferentes em rodovias estaduais do Ceará seriam correlacionáveis ou mostrariam alguma tendência de subestimar ou superestimar a irregularidade. Isto porque em geral um equipamento que usa sensores a laser tem os mesmos acoplados a uma barra que é afixada a um veículo de passeio, tendo largura entre extremos que podem não ser equivalentes às distâncias entre trilhas de roda. A posição convencional é entendida aqui como um sensor colocado num extremo da barra, correspondendo à trilha externa, o segundo localizado no centro e o terceiro no outro extremo da barra, teoricamente fazendo as leituras correspondentes à trilha interna da faixa de tráfego considerada. Para esta avaliação, foram selecionados inicialmente 72 trechos situados na Região Metropolitana de Fortaleza (RMF), e dentre estes foram escolhidos 42, nos quais foram obtidos os valores do IRI com os sensores concentrados do lado direito da barra (LD) e em outra passada, concentrados no lado esquerdo (LE).

A motivação deste estudo se deveu à necessidade de avaliar alguns aspectos da prática brasileira atual nesta questão de avaliação da irregularidade tais como: barras com sensores relativamente curtas, uso de três sensores e uso da média das medidas dos dois sensores extremos na definição da qualidade do pavimento. No exterior é cada vez mais comum as medidas com barras mais longas e com um número maior de sensores visando avaliar com mais detalhes o estado real do pavimento em várias posições intermediárias.

\section{REVISÃO BIBLIOGRÁFICA}

Paterson (1987) cita que um sistema de gerência de pavimentos (SGP) tem por finalidade fundamental fornecer o maior número possível de informações necessárias, para embasar os tomadores de decisão na escolha das melhores estratégias de M\&R e posteriormente analisar e avaliar os resultados. As relações entre parâmetros não devem, necessariamente, resultar de uma análise matemática complexa. O essencial é que elas sejam admitidas e entendidas pela maioria dos usuários do sistema e consideradas como representativas da situação real. O SGP tem como principais metas, proporcionar um atendimento aceitável ao público de modo geral e ser apropriado à estrutura do órgão. Citando-se AASHTO (1990), "Um SGP é projetado como uma ferramenta de planejamento estratégico para a administração superior e como uma ferramenta de engenharia para os tomadores de decisões técnicas”.
Vários estudos realizados nas últimas três décadas indicam que a irregularidade longitudinal é apontada como sendo o parâmetro que mais causava desconforto aos usuários. Carey e Irick (1960) mostraram nos estudos da AASHO, a grande influência que tem a irregularidade longitudinal, na opinião dos motoristas, sobre a qualidade de serventia da superfície dos pavimentos. Alguns defeitos associados com irregularidade foram apontados por Ksaibati et al. (1998): sensação de desconforto ao usuário pela má qualidade de rolamento, menor segurança, ruídos e desgastes dos pneus.

Dada a sua importância na percepção de conforto dos usuários, vários países adotam índices de serventia baseados exclusivamente em medições de irregularidade. Haas e Hudson (1978) destacam que a irregularidade longitudinal não só é uma função do perfil do pavimento, como também é das características do veículo, incluindo pneus, suspensão, etc., assim como dependem da sensibilidade dos usuários quanto às variações de velocidades e de acelerações.

As distorções que originam as irregularidades têm grande influência no custo de operação dos veículos, provocando também neles, os deslocamentos verticais e laterais, originados das respectivas acelerações. O principal desconforto dos usuários, apontado pelos mesmos autores, é decorrente dos deslocamentos verticais originados das distorções longitudinais do perfil do pavimento, que sofrem maior ação das cargas dinâmicas. Por sua vez, os deslocamentos laterais, provenientes também das ações das cargas dinâmicas dos veículos, resultantes de movimentos do veículo para direita e para a esquerda em torno de seu eixo longitudinal, são originados das diferenças das declividades transversais do pavimento e pelas curvas horizontais. Destaca-se ainda a importância da velocidade do veículo no grau de percepção da irregularidade do pavimento pelo usuário.

No Perfil Transversal de um pavimento são verificadas a condição de drenagem superficial da pista e as deformações superficiais plásticas. Estas deformações são originadas da ação do tráfego e se formam normalmente nas trilhas de roda. Elas representam sérios riscos à segurança por facilitar a perda do contato pneu/pavimento, quando o pavimento está molhado, pois em tais deformações pode haver acúmulo de água na pista (Sayers e Karamihas, 1998).

Os Perfis Longitudinais contêm o greide de projeto da rodovia, as irregularidades longitudinais e ainda a textura do pavimento, dependendo da forma e para qual finalidade ele é medido. A condição do pavimento está diretamente relacionada com os dados obtidos na medida deste perfil (Sayers e Karamihas, 1998).

Estudos mostram que 95\% das informações relativas à serventia de pavimentos nos testes originais da 
AASHO estão ligadas exclusivamente à irregularidade do perfil (Haas et al., 1994). A importância da irregularidade tem sido também reconhecida como forma de controle de construção em pavimentos novos. Devido à difusão dos sistemas de gerência de pavimentos ocorrida na última década, tem sido crescente a conscientização nos órgãos públicos da real necessidade de medir as irregularidades longitudinais. Entretanto, verifica-se que há, em vários países, uma grande variação tanto na forma de medir, como nos critérios de aceitação (Farias e Sousa, 2002).

Devido à importância de mensurar as irregularidades longitudinais dos pavimentos, diversos equipamentos podem ser utilizados para esta finalidade. Os equipamentos medidores de irregularidades diferem uns dos outros quanto às características, a produtividade e à eficiência do levantamento. A irregularidade pode ser avaliada por medidores de perfil, tais como: régua, régua deslizante, perfilômetro a laser ou através de medidores tipo resposta - SMITR, mecânicos ou baseado em acelerômetro. Na literatura, vários autores citam os diversos equipamentos ou processos que são utilizados para a medição de parâmetros ligados às irregularidades, dentre eles, DNER (1998); Marcon (1996); Sayers e Karamihas (1998); Farias e Sousa (2002); Haas et al. (1994) e Benevides (2006).

\section{LEVANTAMENTOS}

Para os levantamentos das irregularidades longitudinais nos trechos escolhidos, efetuados em três etapas em junho de 2006 (Benevides, 2006), foi utilizado um aparelho tipo perfilômetro inercial a laser, com três módulos de medição. Este equipamento é constituído de um sistema de medição dos perfis longitudinal e transversal de pavimentos rodoviários, que realiza suas medições com o auxílio de medidores de distância (veículo-pavimento), sem contato (a laser), medidores de aceleração vertical do veículo (acelerômetros), e de um sistema preciso de medição de deslocamento/velocidade. É gerenciado por um sistema micro processado, que coordena a aquisição dos dados e os envia a um computador portátil, em tempo real, através de uma porta do tipo USB. Utiliza o Software Ciberlogger para coleta dos dados e para a determinação dos perfis longitudinais e transversais dos pavimentos, Figura 1 e o Software Cibershell para o cálculo do IRI, tela inicial mostrada na Figura 2, que permite escolher o diretório onde estão gravados e serão processados os dados gerados no campo (Cibermétrica, 2005).

\section{RESULTADOS E MODELOS CORRELACIONANDO IRI NAS 3 POSIÇÕES}

Para a determinação de modelos estatísticos correlacionando os valores do IRI foram utilizados os resultados das medidas efetuadas nos trechos escolhidos da RMF, em junho de 2006, em três etapas (Figura 3):

- A primeira, nos 72 trechos selecionados na RMF, com um total de 493,94 km, com os sensores dispostos na traseira do veículo na posição normal, com distância entre os sensores extremos variando entre 140 e $142 \mathrm{~cm}$ - Tabela 1;

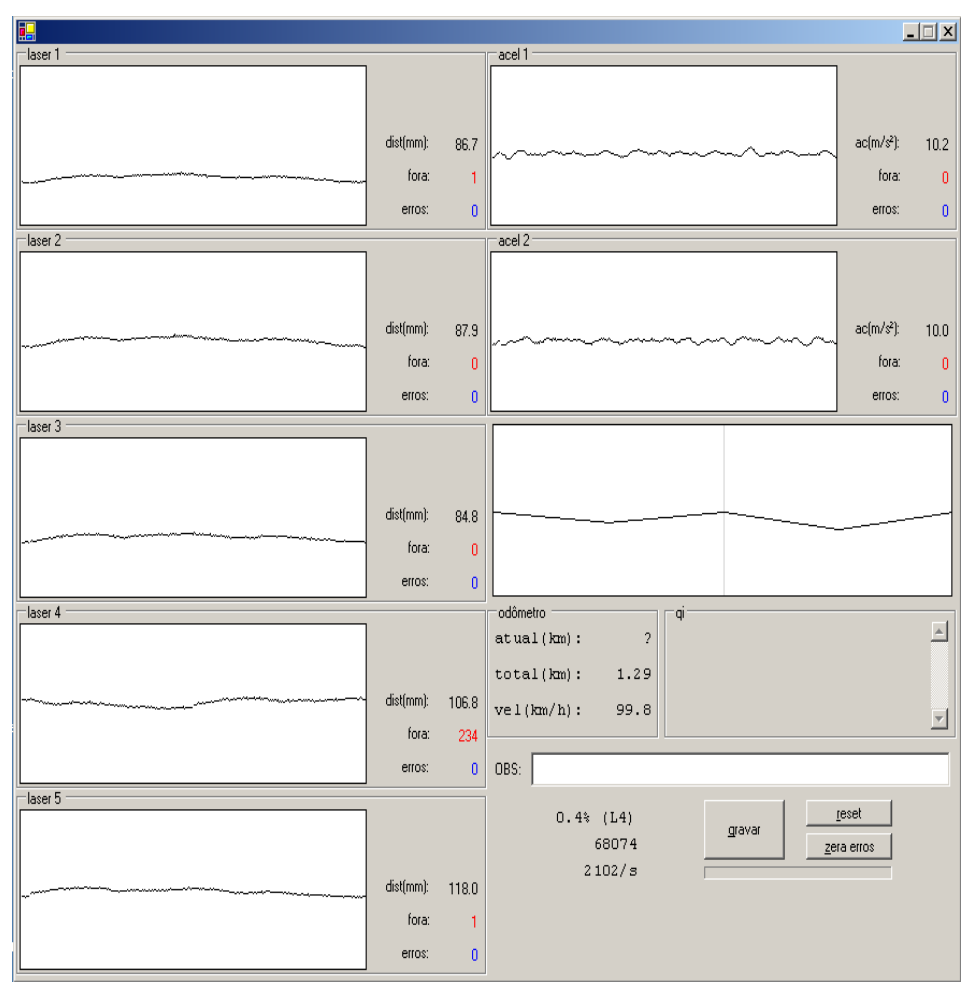

Figura 1. Tela do software Ciberlogger

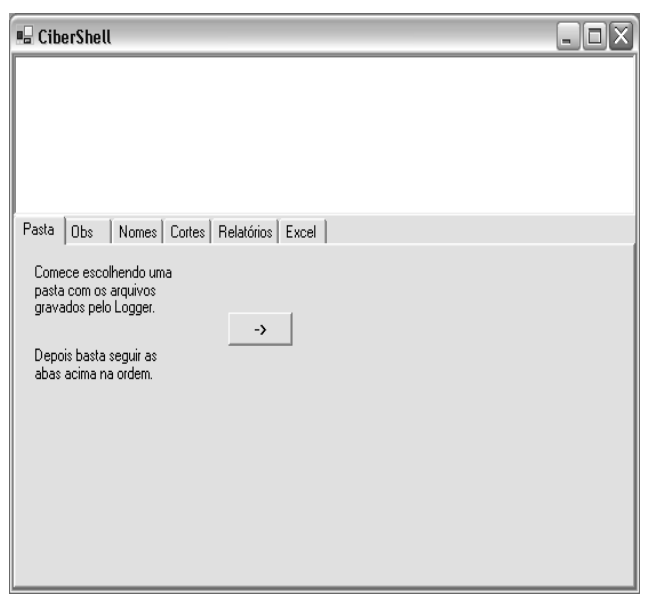

Figura 2. Tela inicial do Cibershell 
- A segunda e a terceira em 42 trechos selecionados dos 72 iniciais, com um total de $333,73 \mathrm{~km}$, com os sensores dispostos na traseira do veículo no lado direito (LD), Figura 4 e lado esquerdo
(LE), Figura 3, com a distância entre os sensores extremos igual a $24 \mathrm{~cm}$ em cada configuração - Tabelas 2 e 3.

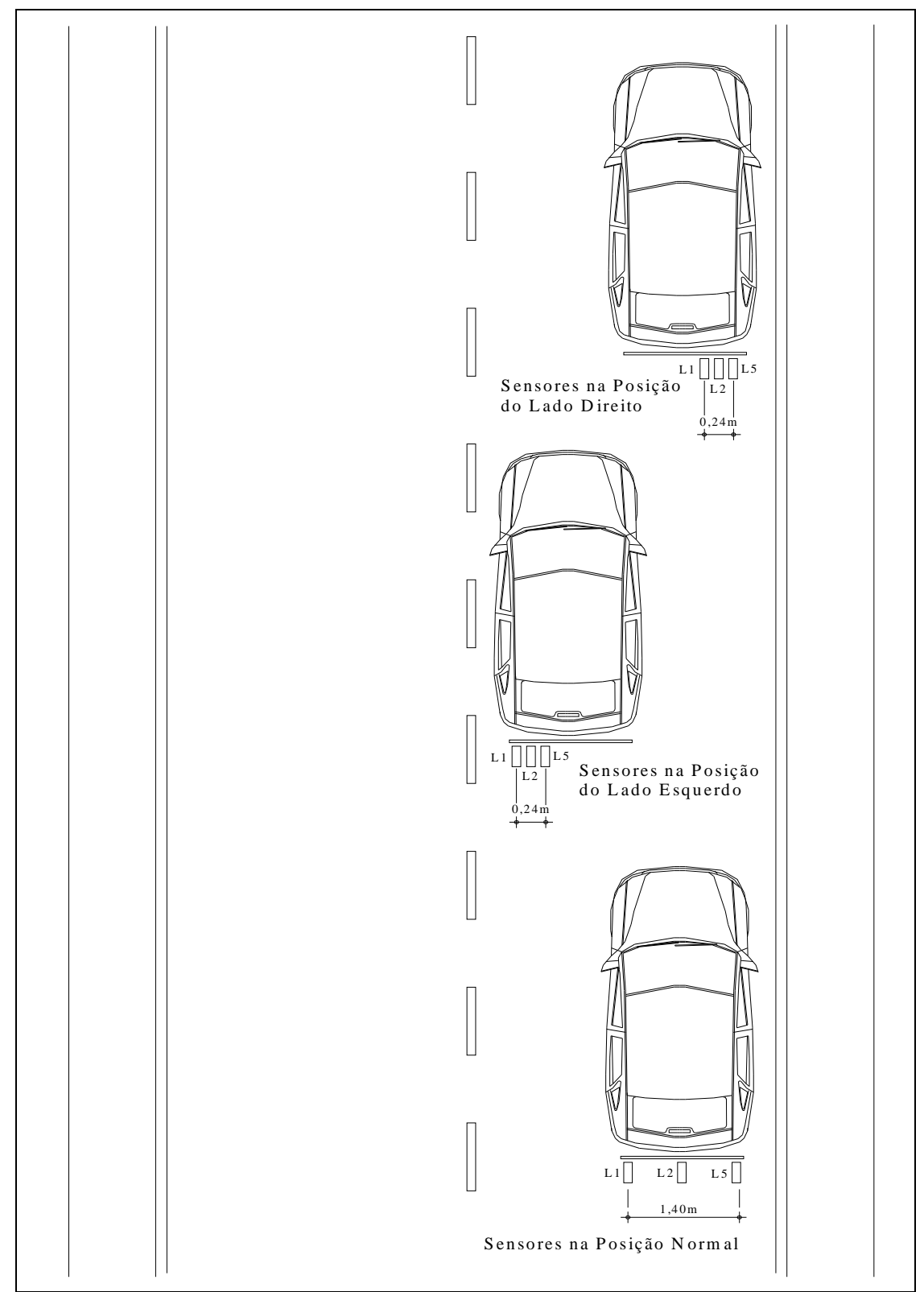

Figura 3. Disposição dos sensores nos levantamentos efetuados para a medição do IRI nesta pesquisa

Tabela 1. Resultados - trilha interna, trilha externa, média e desvio padrão - do IRI dos 72 trechos da RMF, sensores na posição normal (Julho, 2006)

\begin{tabular}{ccccccc}
\hline Trecho & $\begin{array}{c}\text { Extensão } \\
{[\mathbf{m}]}\end{array}$ & $\begin{array}{c}\text { Tipo de } \\
\text { revestimento }\end{array}$ & IRI $_{\text {int }}$ & IRI $_{\text {ext }}$ & IRI $_{\text {médio }}$ & $\begin{array}{c}\text { Desvio padrão } \\
(\boldsymbol{\sigma})\end{array}$ \\
\hline 01-025ECE0010D & 6.335 & CBUQ & 3,66 & 3,53 & 3,60 & 0,09 \\
\hline 02-025ECE0010E & 6.335 & CBUQ & 3,11 & 3,74 & 3,42 & 0,44 \\
\hline 03-025ECE0030D & 6.988 & AAUQ & 4,50 & 4,54 & 4,52 & 0,03 \\
\hline 04-025ECE0030E & 6.988 & AAUQ & 4,37 & 4,22 & 4,30 & 0,11 \\
\hline 05-025ECE0050D & 5.072 & AAUQ & 2,52 & 3,10 & 2,81 & 0,41 \\
\hline 06-025ECE0050E & 5.072 & AAUQ & 2,54 & 2,73 & 2,63 & 0,14 \\
\hline 07-040ECE0010D & 6.940 & CBUQ & 3,19 & 3,46 & 3,33 & 0,19 \\
\hline 08-040ECE0010E & 6.907 & CBUQ & 2,83 & 3,03 & 2,93 & 0,14 \\
\hline 09-040ECE0030D & 2.460 & CBUQ & 2,70 & 3,17 & 2,93 & 0,33 \\
\hline 10-040ECE0030E & 2.451 & CBUQ & 2,37 & 2,86 & 2,62 & 0,35 \\
\hline 11-040ECE0050D & 4.294 & CBUQ & 1,99 & 2,56 & 2,28 & 0,40 \\
\hline 12-040ECE0050E & 4.335 & CBUQ & 2,03 & 2,47 & 2,25 & 0,32 \\
\hline
\end{tabular}


Tabela 1. (cont.) Resultados - trilha interna, trilha externa, média e desvio padrão - do IRI dos 72 trechos da RMF, sensores na posição normal (Julho, 2006)

\begin{tabular}{|c|c|c|c|c|c|c|}
\hline Trecho & $\begin{array}{c}\text { Extensão } \\
{[\mathrm{m}]}\end{array}$ & $\begin{array}{c}\text { Tipo de } \\
\text { revestimento }\end{array}$ & $I_{R} I_{\text {int }}$ & $I_{R} I_{e x t}$ & $I_{R} I_{\text {médio }}$ & $\begin{array}{c}\text { Desvio padrão } \\
(\sigma)\end{array}$ \\
\hline 13-040ECE0070D & 5.310 & CBUQ & 1,79 & 2,16 & 1,98 & 0,26 \\
\hline 14-040ECE0070E & 5.302 & CBUQ & 1,77 & 2,08 & 1,93 & 0,22 \\
\hline 15-040ECE0090D & 3.869 & CBUQ & 2,19 & 2,81 & 2,50 & 0,44 \\
\hline 16-040ECE0090E & 3.907 & CBUQ & 2,08 & 2,30 & 2,19 & 0,15 \\
\hline 17-040ECE0110D & 2.533 & CBUQ & 2,41 & 3,00 & 2,71 & 0,42 \\
\hline 18-040ECE0110E & 2.511 & CBUQ & 2,71 & 2,80 & 2,75 & 0,07 \\
\hline 19-040ECE0130D & 6.488 & CBUQ & 2,09 & 2,42 & 2,26 & 0,23 \\
\hline 20-040ECE0130E & 6.488 & CBUQ & 2,34 & 2,20 & 2,27 & 0,10 \\
\hline 21-040ECE0150D & 9.131 & CBUQ & 2,30 & 2,51 & 2,41 & 0,15 \\
\hline 22-040ECE0150E & 9.131 & CBUQ & 2,24 & 2,28 & 2,26 & 0,02 \\
\hline 23-040ECE0170D & 9.940 & CBUQ & 2,46 & 2,35 & 2,41 & 0,08 \\
\hline 24-040ECE0170E & 9.940 & CBUQ & 2,40 & 2,15 & 2,27 & 0,18 \\
\hline 25-060ECE0010D & 3.838 & CBUQ & 3,40 & 3,63 & 3,51 & 0,17 \\
\hline 26-060ECE0010E & 3.829 & CBUQ & 3,09 & 3,01 & 3,05 & 0,06 \\
\hline 27-060ECE0030D & 3.806 & CBUQ & 3,99 & 3,59 & 3,79 & 0,29 \\
\hline 28-060ECE0030E & 3.778 & CBUQ & 2,91 & 3,08 & 2,99 & 0,12 \\
\hline 29-060ECE0050D & 5.809 & CBUQ & 3,17 & 3,02 & 3,10 & 0,10 \\
\hline 30-060ECE0050E & 5.819 & CBUQ & 3,02 & 2,89 & 2,96 & 0,09 \\
\hline 31-060ECE0070D & 6.257 & CBUQ & 2,89 & 2,89 & 2,89 & 0,00 \\
\hline 32-060ECE0070E & 6.204 & CBUQ & 2,62 & 2,65 & 2,63 & 0,02 \\
\hline 33-060ECE0090D & 1.950 & CBUQ & 3,09 & 3,31 & 3,20 & 0,16 \\
\hline 34-060ECE0090E & 1.974 & CBUQ & 2,92 & 3,08 & 3,00 & 0,11 \\
\hline 35-060ECE0110D & 5.267 & CBUQ & 2,97 & 2,89 & 2,93 & 0,06 \\
\hline 36-060ECE0110E & 5.267 & CBUQ & 2,92 & 3,23 & 3,08 & 0,21 \\
\hline 37-060ECE0130D & 15.865 & CBUQ & 2,57 & 2,17 & 2,37 & 0,28 \\
\hline 38-060ECE0130E & 15.865 & CBUQ & 2,64 & 2,58 & 2,61 & 0,04 \\
\hline 39-060ECE0150D & 6.921 & CBUQ & 2,78 & 2,60 & 2,69 & 0,13 \\
\hline 40-060ECE0150E & 6.921 & CBUQ & 2,98 & 3,07 & 3,03 & 0,07 \\
\hline 41-065ECE0010D & 316 & CBUQ & 5,77 & 6,36 & 6,07 & 0,42 \\
\hline 42-065ECE0010E & 316 & CBUQ & 6,72 & 8,63 & 7,67 & 1,35 \\
\hline 43-065ECE0030D & 3.564 & CBUQ & 2,33 & 2,67 & 2,50 & 0,24 \\
\hline 44-065ECE0030E & 3.597 & CBUQ & 2,03 & 2,30 & 2,17 & 0,20 \\
\hline 45-065ECE0050D & 6.835 & CBUQ & 2,19 & 2,25 & 2,22 & 0,04 \\
\hline 46-065ECE0050E & 6.861 & CBUQ & 2,07 & 2,20 & 2,13 & 0,09 \\
\hline 47-085ECE0030D & 1.483 & TSD & 4,59 & 4,92 & 4,76 & 0,23 \\
\hline 48-085ECE0030E & 1.483 & TSD & 5,66 & 5,57 & 5,61 & 0,07 \\
\hline 49-085ECE0050D & 1.956 & TSD & 3,67 & 3,64 & 3,65 & 0,01 \\
\hline 50-085ECE0050E & 1.956 & TSD & 3,97 & 4,41 & 4,19 & 0,31 \\
\hline 51-085ECE0070D & 14.728 & TSD & 3,31 & 3,57 & 3,44 & 0,18 \\
\hline 52-085ECE0070E & 14.728 & TSD & 3,36 & 3,80 & 3,58 & 0,31 \\
\hline 53-085ECE0090D & 8.337 & TSD & 3,16 & 3,46 & 3,31 & 0,22 \\
\hline 54-085ECE0090E & 8.337 & TSD & 3,32 & 3,88 & 3,60 & 0,40 \\
\hline 55-085ECE0110D & 4.392 & TSD & 3,21 & 3,39 & 3,30 & 0,13 \\
\hline 56-085ECE0110E & 4.392 & TSD & 3,39 & 3,56 & 3,47 & 0,12 \\
\hline 57-085ECE0130D & 6.628 & TSD & 3,12 & 3,21 & 3,17 & 0,06 \\
\hline 58-085ECE0130E & 6.628 & TSD & 2,92 & 3,48 & 3,20 & 0,40 \\
\hline 59-085ECE0150D & 8.658 & TSD & 3,04 & 3,29 & 3,17 & 0,18 \\
\hline 60-085ECE0150E & 8.658 & TSD & 3,31 & 4,44 & 3,88 & 0,80 \\
\hline 61-085ECE0170D & 14.291 & TSD & 2,98 & 3,07 & 3,03 & 0,06 \\
\hline 62-085ECE0170E & 14.291 & TSD & 3,12 & 3,26 & 3,19 & 0,10 \\
\hline 63-090ECE0010D & 7.244 & TSD & 4,32 & 4,61 & 4,46 & 0,20 \\
\hline 64-090ECE0010E & 7.244 & TSD & 4,32 & 4,61 & 4,46 & 0,20 \\
\hline 65-341ECE0010D & 11.561 & AAUQ & 3,15 & 3,12 & 3,14 & 0,02 \\
\hline 66-341ECE0010E & 11.561 & AAUQ & 2,87 & 2,94 & 2,91 & 0,04 \\
\hline 67-341ECE0030D & 4.434 & AAUQ & 2,25 & 2,11 & 2,18 & 0,10 \\
\hline 68-341ECE0030E & 4.434 & AAUQ & 2,34 & 2,35 & 2,35 & 0,00 \\
\hline 69-341ECE0050D & 12.565 & AAUQ & 2,28 & 2,03 & 2,16 & 0,18 \\
\hline 70-341ECE0050E & 12.565 & AAUQ & 2,26 & 2,22 & 2,24 & 0,03 \\
\hline 71-341ECE0070D & 20.903 & TSD & 2,55 & 2,45 & 2,50 & 0,07 \\
\hline 72-341ECE0070E & 20.903 & TSD & 2,69 & 2,39 & 2,54 & 0,21 \\
\hline
\end{tabular}




\subsection{Modelo correlacionando o resultado do IRI nas trilhas interna e externa, com a localização dos sensores na posição normal.}

Para a geração deste modelo foram considerados os resultados do IRI $I_{\text {ext }} \times \operatorname{IRI}_{\text {int }}$ da Tabela 1 para os 72 trechos escolhidos. Foram estabelecidas equações de regressão sendo usados os programas Microsoft Office Excel e SPSS (Statistical Package for the Social Sciences), utilizando a Análise da Variância (ANOVA). $\mathrm{Na}$ análise estatística da equação de regressão e planilhas de cálculo, mostradas na Tabela 4 para este modelo, verifica-se que o valor de $F, 645,13$ é maior do que $\mathrm{F}_{0,05,1,70}=3,84$, valor crítico, e que o valor- $P$ da estatística t é inferior a 2,5\%. Portanto, o modelo é significante. Na Figura 4 mostram-se pontos e equação ajustada. Verifica-se que à medida que o IRI vai aumentando, é maior a diferença de valores entre as trilhas, preponderando os maiores valores na trilha externa ou do lado direito da pista. Esta assertiva concorda com resultados de outros pesquisadores, dentre eles Ksaibati et al., (1998).

\subsection{Modelo correlacionando os resultados do IRI médio, com a localização dos três sensores no lado direito (LD) e no lado esquerdo (LE)}

Foram utilizados neste modelo os valores do IRI médio da Tabela 2, com os três sensores no LD e da Tabela 3 com os três sensores no LE, nos 42 trechos selecionados da RMF.

Tabela 2. Resultados do IRI com os três sensores no LCD nos 42 trechos da RMF (2006)

\begin{tabular}{|c|c|c|c|c|c|c|}
\hline Trecho & $\begin{array}{c}\text { Extensão } \\
\text { [m] }\end{array}$ & $\begin{array}{c}\text { Tipo de } \\
\text { revestimento }\end{array}$ & $I R I_{\text {int }}$ & $\operatorname{IRI}_{C}$ & $\operatorname{IRI}_{\text {ext }}$ & $I R I_{\text {médio }}$ \\
\hline 25-060ECE0010D & 3.838 & CBUQ & 3,52 & 3,57 & 3,65 & 3,58 \\
\hline 26-060ECE0010E & 3.829 & CBUQ & 2,75 & 2,79 & 2,83 & 2,79 \\
\hline 27-060ECE0030D & 3.806 & CBUQ & 3,43 & 3,60 & 3,82 & 3,62 \\
\hline 28-060ECE0030E & 3.778 & CBUQ & 2,89 & 3,08 & 3,21 & 3,06 \\
\hline 29-060ECE0050D & 5.809 & CBUQ & 2,87 & 2,92 & 2,98 & 2,93 \\
\hline 30-060ECE0050E & 5.819 & CBUQ & 2,84 & 2,89 & 2,95 & 2,89 \\
\hline 31-060ECE0070D & 6.257 & CBUQ & 2,86 & 2,86 & 2,87 & 2,86 \\
\hline 32-060ECE0070E & 6.204 & CBUQ & 2,68 & 2,71 & 2,74 & 2,71 \\
\hline 33-060ECE0090D & 1.950 & CBUQ & 3,02 & 3,10 & 3,22 & 3,11 \\
\hline 34-060ECE0090E & 1.974 & CBUQ & 3,18 & 3,22 & 3,27 & 3,22 \\
\hline 35-060ECE0110D & 5.267 & CBUQ & 2,64 & 2,78 & 2,93 & 2,78 \\
\hline 36-060ECE0110E & 5.267 & CBUQ & 3,10 & 3,24 & 3,38 & 3,24 \\
\hline 37-060ECE0130D & 15.865 & CBUQ & 2,09 & 2,15 & 2,25 & 2,16 \\
\hline 38-060ECE0130E & 15.865 & CBUQ & 2,43 & 2,56 & 2,67 & 2,55 \\
\hline 39-060ECE0150D & 6.921 & CBUQ & 2,44 & 2,59 & 2,70 & 2,58 \\
\hline 40-060ECE0150E & 6.921 & CBUQ & 2,78 & 2,97 & 3,19 & 2,98 \\
\hline 47-085ECE0030D & 1.483 & TSD & 4,92 & 5,22 & 5,33 & 5,16 \\
\hline 48-085ECE0030E & 1.483 & TSD & 5,40 & 5,81 & 5,95 & 5,72 \\
\hline 49-085ECE0050D & 1.956 & TSD & 3,59 & 3,65 & 3,78 & 3,67 \\
\hline 50-085ECE0050E & 1.956 & TSD & 4,89 & 5,02 & 5,23 & 5,05 \\
\hline 51-085ECE0070D & 14.728 & TSD & 3,45 & 3,56 & 3,67 & 3,56 \\
\hline 52-085ECE0070E & 14.728 & TSD & 3,66 & 3,75 & 3,83 & 3,75 \\
\hline 53-085ECE0090D & 8.337 & TSD & 3,39 & 3,41 & 3,52 & 3,44 \\
\hline 54-085ECE0090E & 8.337 & TSD & 3,36 & 3,41 & 3,49 & 3,42 \\
\hline 55-085ECE0110D & 4.392 & TSD & 3,60 & 3,63 & 3,66 & 3,63 \\
\hline 56-085ECE0110E & 4.392 & TSD & 3,42 & 3,43 & 3,50 & 3,45 \\
\hline 57-085ECE0130D & 6.628 & TSD & 3,48 & 3,40 & 3,38 & 3,42 \\
\hline 58-085ECE0130E & 6.628 & TSD & 3,32 & 3,32 & 3,32 & 3,32 \\
\hline 59-085ECE0150D & 8.658 & TSD & 3,40 & 3,40 & 3,50 & 3,43 \\
\hline 60-085ECE0150E & 8.658 & TSD & 3,69 & 3,74 & 3,84 & 3,76 \\
\hline 61-085ECE0170D & 14.291 & TSD & 3,02 & 3,02 & 3,08 & 3,04 \\
\hline 62-085ECE0170E & 14.291 & TSD & 3,13 & 3,18 & 3,22 & 3,17 \\
\hline 63-090ECE0010D & 7.244 & TSD & 4,59 & 4,87 & 5,08 & 4,85 \\
\hline 64-090ECE0010E & 7.244 & TSD & 3,78 & 3,87 & 3,97 & 3,87 \\
\hline 65-341ECE0010D & 11.561 & AAUQ & 2,97 & 3,02 & 3,10 & 3,03 \\
\hline 66-341ECE0010E & 11.561 & AAUQ & 2,86 & 2,94 & 3,01 & 2,94 \\
\hline 67-341ECE0030D & 4.434 & AAUQ & 2,05 & 2,15 & 2,25 & 2,15 \\
\hline 68-341ECE0030E & 4.434 & AAUQ & 2,20 & 2,24 & 2,29 & 2,25 \\
\hline 69-341ECE0050D & 12.565 & AAUQ & 1,93 & 2,00 & 2,08 & 2,00 \\
\hline 70-341ECE0050E & 12.565 & AAUQ & 2,06 & 2,13 & 2,23 & 2,14 \\
\hline 71-341ECE0070D & 20.903 & TSD & 2,43 & 2,46 & 2,56 & 2,48 \\
\hline 72-341ECE0070E & 20.903 & TSD & 2,32 & 2,33 & 2,39 & 2,35 \\
\hline
\end{tabular}


Na análise estatística do modelo, observa-se que o teste de Snedecor é atendido, o valor de $F=498,48$ é maior que $\mathrm{F}_{0,05,1,40}=4,08$, assim como valor- $P$ da estatística t é inferior a 2,5\%.

Os resumos estatísticos deste modelo estão em Benevides (2006). Na Figura 5 está apresentado o gráfico com a equação de regressão e com o $\mathrm{R}^{2}$ do modelo para os 42 trechos da RMF. Verifica-se, na análise deste modelo, a mesma tendência observada nos modelos anteriores, ou seja, preponderam os maiores valores do IRI médio para a disposição dos três sensores no lado direito, quando estes valores vão aumentando.

\subsection{Modelo correlacionando o IRI $\left.\right|_{\text {int }}$ com os sensores na posição normal e o IRI ${ }_{\text {int }}$}

\section{com os sensores no lado esquerdo}

Neste modelo foram utilizados os resultados do IRI $_{\text {int }}$ da Tabela 3 para os 42 trechos com os sensores dispostos no lado esquerdo e da Tabela 1 para os mesmos trechos para os sensores dispostos na posição normal. Verifica-se que são atendidos os critérios analisados nos modelos anteriores: $F=347,29$ é maior que $\mathrm{F}_{0,05,1}$, ${ }_{40}=4,08$, assim como o valor- $P$ da estatística t é inferior a 2,5\%. Detalhes deste modelo estão em Benevides (2006). Na Figura 6 mostram-se os pontos e a equação de correlação. Observa-se forte correlação entre os valores do IRI $I_{\text {int }}$ para as citadas disposições dos sensores, ao analisar a equação e o coeficiente de determinação $\mathrm{R}^{2}$ do modelo

Tabela 3. Resultados do IRI com três sensores no LE nos 42 trechos da RMF (2006)

\begin{tabular}{|c|c|c|c|c|c|c|}
\hline Trecho & $\begin{array}{c}\text { Extensão } \\
{[\mathrm{m}]}\end{array}$ & $\begin{array}{c}\text { Tipo de } \\
\text { revestimento }\end{array}$ & $I R I_{\text {int }}$ & $\operatorname{IRI}_{C}$ & $I R I_{e x t}$ & $I R I_{\text {médio }}$ \\
\hline 25-060ECE0010D & 3.838 & CBUQ & 3,51 & 3,49 & 3,51 & 3,50 \\
\hline 26-060ECE0010E & 3.829 & CBUQ & 3,00 & 2,96 & 2,95 & 2,97 \\
\hline 27-060ECE0030D & 3.806 & CBUQ & 3,60 & 3,49 & 3,49 & 3,53 \\
\hline 28-060ECE0030E & 3.778 & CBUQ & 3,09 & 3,11 & 3,13 & 3,11 \\
\hline 29-060ECE0050D & 5.809 & CBUQ & 3,13 & 3,12 & 3,10 & 3,12 \\
\hline 30-060ECE0050E & 5.819 & CBUQ & 3,32 & 3,08 & 3,14 & 3,18 \\
\hline 31-060ECE0070D & 6.257 & CBUQ & 2,96 & 2,94 & 2,95 & 2,95 \\
\hline 32-060ECE0070E & 6.204 & CBUQ & 2,74 & 2,62 & 2,63 & 2,66 \\
\hline 33-060ECE0090D & 1.950 & CBUQ & 3,01 & 2,97 & 2,98 & 2,99 \\
\hline 34-060ECE0090E & 1.974 & CBUQ & 3,35 & 3,06 & 3,11 & 3,17 \\
\hline 35-060ECE0110D & 5.267 & CBUQ & 2,90 & 2,84 & 2,86 & 2,87 \\
\hline 36-060ECE0110E & 5.267 & CBUQ & 2,95 & 2,89 & 2,87 & 2,90 \\
\hline 37-060ECE0130D & 15.865 & CBUQ & 2,46 & 2,41 & 2,38 & 2,42 \\
\hline 38-060ECE0130E & 15.865 & CBUQ & 2,65 & 2,60 & 2,58 & 2,61 \\
\hline 39-060ECE0150D & 6.921 & CBUQ & 2,77 & 2,72 & 2,70 & 2,73 \\
\hline 40-060ECE0150E & 6.921 & CBUQ & 2,88 & 2,83 & 2,81 & 2,84 \\
\hline 47-085ECE0030D & 1.483 & TSD & 4,83 & 4,86 & 4,85 & 4,84 \\
\hline 48-085ECE0030E & 1.483 & TSD & 4,82 & 4,77 & 4,84 & 4,81 \\
\hline 49-085ECE0050D & 1.956 & TSD & 3,76 & 3,73 & 3,65 & 3,71 \\
\hline 50-085ECE0050E & 1.956 & TSD & 4,58 & 4,56 & 4,58 & 4,57 \\
\hline 51-085ECE0070D & 14.728 & TSD & 3,33 & 3,34 & 3,37 & 3,35 \\
\hline 52-085ECE0070E & 14.728 & TSD & 3,37 & 3,36 & 3,39 & 3,37 \\
\hline 53-085ECE0090D & 8.337 & TSD & 3,23 & 3,24 & 3,27 & 3,25 \\
\hline 54-085ECE0090E & 8.337 & TSD & 3,22 & 3,26 & 3,37 & 3,28 \\
\hline 55-085ECE0110D & 4.392 & TSD & 3,25 & 3,30 & 3,38 & 3,31 \\
\hline 56-085ECE0110E & 4.392 & TSD & 3,36 & 3,38 & 3,36 & 3,37 \\
\hline 57-085ECE0130D & 6.628 & TSD & 3,15 & 3,19 & 3,22 & 3,19 \\
\hline 58-085ECE0130E & 6.628 & TSD & 3,05 & 3,07 & 3,12 & 3,08 \\
\hline 59-085ECE0150D & 8.658 & TSD & 3,06 & 3,12 & 3,14 & 3,10 \\
\hline 60-085ECE0150E & 8.658 & TSD & 3,06 & 3,12 & 3,14 & 3,10 \\
\hline 61-085ECE0170D & 14.291 & TSD & 2,85 & 2,88 & 2,92 & 2,89 \\
\hline 62-085ECE0170E & 14.291 & TSD & 2,97 & 2,96 & 2,99 & 2,98 \\
\hline 63-090ECE0010D & 7.244 & TSD & 4,67 & 4,56 & 4,42 & 4,55 \\
\hline 64-090ECE0010E & 7.244 & TSD & 4,40 & 4,25 & 4,10 & 4,25 \\
\hline 65-341ECE0010D & 11.561 & AAUQ & 2,98 & 3,04 & 3,08 & 3,03 \\
\hline 66-341ECE0010E & 11.561 & AAUQ & 2,85 & 2,88 & 2,92 & 2,88 \\
\hline 67-341ECE0030D & 4.434 & AAUQ & 2,49 & 2,43 & 2,33 & 2,42 \\
\hline 68-341ECE0030E & 4.434 & AAUQ & 2,33 & 2,32 & 2,26 & 2,30 \\
\hline 69-341ECE0050D & 12.565 & AAUQ & 2,32 & 2,27 & 2,22 & 2,27 \\
\hline 70-341ECE0050E & 12.565 & AAUQ & 2,20 & 2,17 & 2,10 & 2,16 \\
\hline 71-341ECE0070D & 20.903 & TSD & 2,47 & 2,48 & 2,47 & 2,47 \\
\hline 72-341ECE0070E & 20.903 & TSD & 2,57 & 2,54 & 2,54 & 2,55 \\
\hline
\end{tabular}


Tabela 4. Resumo dos resultados do modelo IRI $I_{\text {int }} \times I_{\mathrm{RI}} \mathrm{Ixt}_{\text {para }} \mathrm{SPN}$ nos 72 trechos

\section{Estatística de regressão}

\begin{tabular}{lc}
\hline R múltiplo & 0,949797805 \\
R-Quadrado & 0,90211587 \\
R-quadrado ajustado & 0,900717526 \\
Erro padrão & 0,287450225 \\
Observações & 72 \\
\hline
\end{tabular}

Observações 72

\begin{tabular}{|c|c|c|c|c|c|c|}
\hline \multicolumn{7}{|c|}{ ANOVA } \\
\hline & & & $g l$ & $S Q$ & $M Q$ & $F$ \\
\hline Regressão & & & 1 & 53,30566738 & 53,3056674 & 645,131 \\
\hline Resíduo & & & 70 & 5,78393422 & 0,08262763 & \\
\hline \multirow[t]{2}{*}{ Total } & & & 71 & 59,0896016 & & \\
\hline & Coeficientes & Erro padrão & Stat $t$ & Valor P & $\begin{array}{c}95 \% \\
\text { inferiores }\end{array}$ & $\begin{array}{c}95 \% \\
\text { superiores }\end{array}$ \\
\hline Interseção & 0,412224407 & 0,107812325 & 3,82353695 & 0,00028 & 0,1972 & 0,627249 \\
\hline Variável X1 & 0,81239461 & 0,031984752 & 25,3994341 & 4,7E-37 & 0,748603 & 0,876186 \\
\hline
\end{tabular}

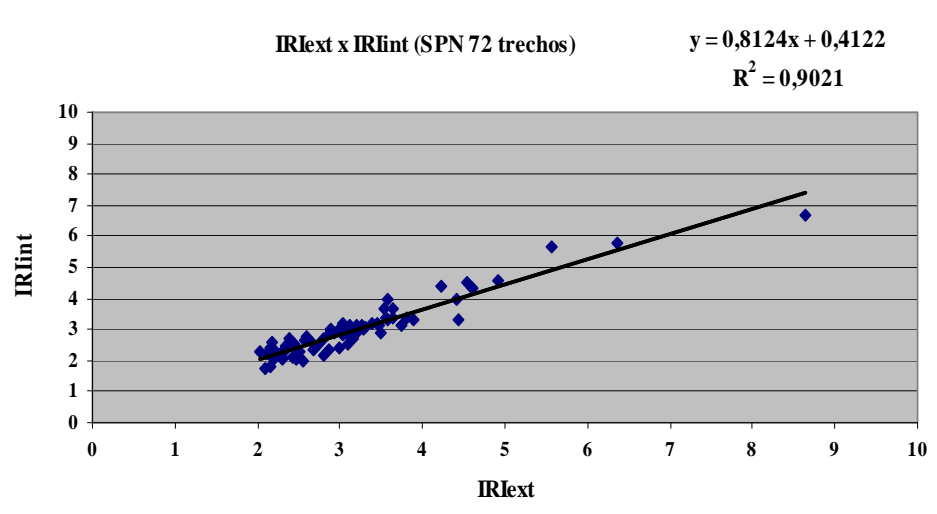

Figura 4. Modelo $I R I_{\text {int }} \times I R I_{\text {ext }}$ para SPN nos 72 trechos da RMF

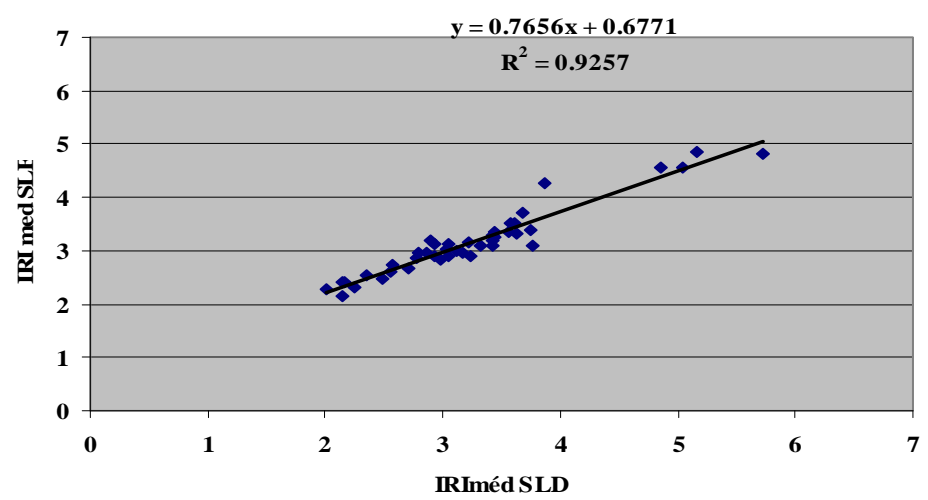

Figura 5. Modelo IRI médio $S L D \times I R I_{\text {médio }}$ SLE em 42 trechos desta pesquisa

Verifica-se na análise estatística dos três modelos de correlação entre os valores do IRI medidos com o perfilômetro a laser na Tabela 7 , que os valores de $F$ (teste de Snedecor) são sempre maiores do que o $F$ crítico. Portanto, rejeita-se a hipótese nula e existe uma confiança de $95 \%$ de aceitação da significância dos modelos.

Verifica-se na análise das equações que correlacionam os resultados do IRI nas trilhas interna e externa, IRI $_{\text {int }} \times$ IRI $_{\text {ext }}$, obtidos com a localização dos sensores na posição normal, altos valores do coeficiente de determinação $\left(\mathrm{R}^{2}\right)$. Observa-se também que à medida que o valor do IRI vai aumentando, torna-se maior a diferença de valores do IRI entre as trilhas, preponde- rando os maiores valores na trilha externa ou seja os medidos do lado direito. No modelo correlacionando os resultados do IRI médio, obtidos com a localização dos três sensores no lado direito (LD) e no lado esquerdo (LE), também com um alto valor do $\mathrm{R}^{2}$, observa-se a mesma tendência do modelo anterior. Preponderam os maiores valores do IRI médio para a disposição dos três sensores no lado direito, quando estes valores vão aumentando. No modelo de correlação entre os valores do $\mathrm{IRI}_{\text {int }}$ com os sensores na posição

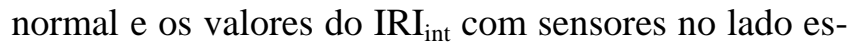
querdo, que apresenta bom valor de $\mathrm{R}^{2}$. 


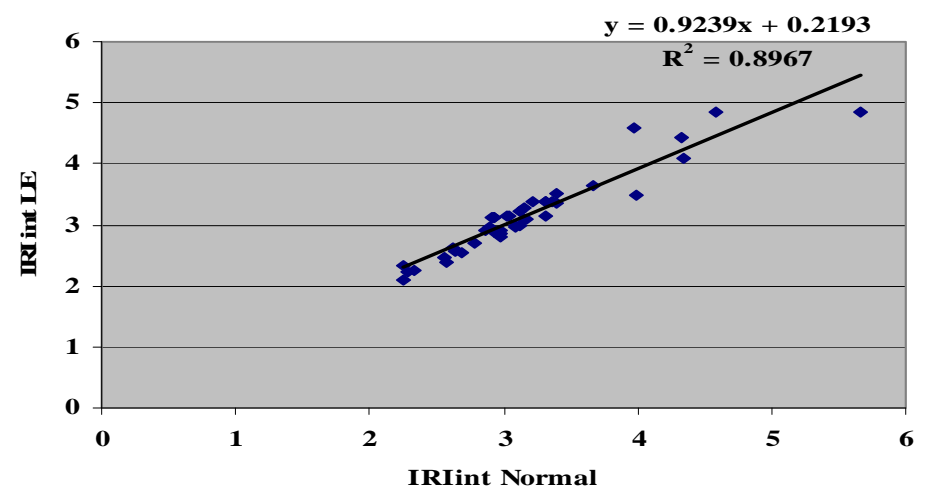

Figura 6. Modelo IRI $\left.\right|_{\text {int }} \mathrm{SPN} \times \mathrm{IR} \mathrm{I}_{\text {int }} \mathrm{SLE}$ em 42 trechos desta pesquisa

Tabela 7. Resultado da análise dos modelos de correlação entre valores do IRI medidos com o perfilômetro a laser em função de $F$

\begin{tabular}{cllll}
\hline $\begin{array}{c}\text { Valores de IRI } \\
\text { comparados }\end{array}$ & \multicolumn{1}{c}{ Modelo } & F & $\boldsymbol{F}_{\text {crítico }}$ & Resultado \\
\hline $\begin{array}{c}\left(\mathrm{IRI}_{\mathrm{I}} \times \mathrm{IRI}_{\mathrm{E}}\right) \mathrm{SPN} \\
72 \text { trechos }\end{array}$ & $\begin{array}{l}y=0,8124 \cdot x+0,4122 \\
R^{2}=0,9021\end{array}$ & 645,13 & 3,84 & Significativo \\
\hline $\mathrm{IRI}_{\text {médio }}(\mathrm{SLD} \times \mathrm{SLE})$ & $\begin{array}{l}y=0,7656 \cdot x+0,6771 \\
42 \text { trechos }\end{array}$ & 498,48 & 4,08 & Significativo \\
\hline$R^{2}=0,9257$ & $\begin{array}{l}y=0,9239 \cdot x+0,2193 \\
\mathrm{IRI}_{\text {int }}(\mathrm{SPN} \times \mathrm{SLE}) \\
42 \text { trechos }\end{array}$ & 347,29 & 4,08 & Significativo \\
\hline
\end{tabular}

\section{CONSIDERAÇÕES FINAIS}

Embora haja forte correlação entre os valores medidos nas três disposições dos sensores laser na barra de fixação ao veículo, constatam-se diferenças entre estes valores. Isto é mais sensível quando se comparam os valores da trilha externa em relação à trilha interna, $\mathrm{e}$ tem como era esperado, maior efeito quanto maior for à irregularidade. Eventualmente é possível ter avaliações de qualidade diferentes no mesmo trecho nos casos em que as características de irregularidade estejam muito próximas aos limites de aceitação por critério. Deve-se discutir o critério correntemente adotado de admitir valor médio entre trilhas na avaliação das características de um trecho quanto ao conforto de usuário, visto que frequentemente há diferenças sensíveis entre as duas trilhas e a média mascara a gravidade da situação da irregularidade real. Medições de IRI têm a influência da utilização da haste de 1,40 m, assim como do espaçamento entre os sensores e o posicionamento dos mesmos. É urgente normatizar estas medidas no Brasil, bem como criar norma de calibração de equipamentos de IRI por sensores a laser. É necessário também definir número mínimo de sensores.

\section{REFERÊNCIAS BIBLIOGRÁFICAS}

AASHTO (1990) Guide for Design Management Systems. American Association of State Highway and Transportation Officials, Washington DC.

Benevides, S.A. S. (2006) Modelos de desempenho de pavimentos asfálticos para um sistema de gestão de rodovias estaduais do Ceará Tese de Doutorado em ciências em Engenharia Civil, COPPE/UFRJ.
Carey JR., W.N. e Irick, P.E. (1960) The Pavement Serviciability Performance Concept. In: Highway Research Board - Buletin 250, p.4058.

Cibermétrica, (2005), Manual de Instalação e Operação do Perfilômetro Laser para Pavimentos.

DNER, (1998) Manual de Reabilitação de Pavimentos Asfálticos. IPR/DNER/ABNT Publ. 704, 1 ed.

Farias, M. M. \& Souza, R. O., (2002), Irregularidade Longitudinal e sua Influência na Avaliação Funcional de Pavimentos. In: VII Encontro Nacional de Conservação Rodoviária, Vitória, ES. CD Rom.

Haas, R., Hudson, W.R. \& Zaniewskij, J., (1994), Modern Pavement Management, Malabar/Florida, Krieger.

Haas, R., Hudson, W. R., 1978, Pavement Management Systems. EUA: McGraw-Hill.

Ksaibati, K., McNamara, R. \& Armaghani, J., (1998), A Comparison of Roughness Measurement from Laser and Ultrasonic Road Profilers. In: Research Report FL/DOT/SMO/98-425, STATE OF FLORIDA.

Medina, J., 1997, Mecânica dos Pavimentos, $1^{\text {a }}$ ed. Rio de Janeiro, COPPE/UFRJ.

Paterson, W.D.O., (1987), Road Deterioration and Maintenance Effects Models for Planning and Management. The World Bank, Baltimore. The John Hopkins University Press.

Sayers, M. W. e Karamihas, S. M., (1998), The Little Book of Profiling. Basic Information about Measuring and Interpreting Road Profiles. The Regent of the University of Michigan, $102 \mathrm{p}$.

SHRP, (1993), Distress Identification Manual for the Long Term Pavement Performance Project. Strategic Highway Research Program, National Academy of Sciences, Washington, D C. 